\title{
A Two Tier QoS Provisioning Scheme for Satellite based Multimedia Communication
}

\author{
Sorum Kotia \\ PhD Scholar, \\ EC Department \\ Nirma University \\ Ahmedabad, India
}

\author{
Dr. K.S.Dasgupta \\ Director \\ Indian Institute of Space \\ Science \& Technology \\ Thiruvananthapuram, India
}

\author{
Dr. M.D.Desai \\ Professor, \\ EC Department, \\ Kalol Institute of Technology \\ Ahmedabad, India
}

\begin{abstract}
This paper suggests a generic scheme for provisioning of QoS under largely fluctuating channel quality and flat fading conditions for multimedia communication over satellite. A resolution scalable source is matched to a hierarchical constellation in order to achieve an adaptive decoding capability to combat fluctuations in signal to noise ratio (SNR). The receiver estimates the SNR and if the SNR is found to be below a certain threshold, it reduces the rate by dropping off a higher order constellation which is riding on a baseline QPSK constellation. Thus, the first tier of the scheme guarantees service at lower resolution in poor channel conditions. The second tier of the scheme facilitates near coherent demodulation under frequency nonselective fading conditions. It makes use of pilot symbols which are embedded in the information symbols to probe the fading channel. The received pilots provide an estimate of multiplicative distortion encountered in slow \& flat fading channel. This estimated amplitude and phase reference are used to adjust the level and angle of the incoming symbols to the reference constellation. This receiver based rate adaptation scheme is typically suitable for broadcast applications and addresses the issue of service quality provision for a multitude of receivers. It provides a stable reference for demodulation under fading and supports graceful degradation for deteriorating channel conditions. Exhaustive simulations are carried out to examine the validity of the concept.
\end{abstract}

\section{General Terms}

Joint source and channel coding (JSCC)

\section{Keywords}

Resolution scalability, Unequal error protection, Hierarchical constellation, Pilot symbol assisted modulation

\section{INTRODUCTION}

There is an ever increasing demand of anytime, anywhere exchange of all type of information over a variety of handheld, mobile and stationary terminals. The existing communication infrastructure needs to evolve in a manner so that it can keep pace with this multimedia revolution. Satellite technology when considered in conjunction with existing communication systems may offer the necessary augmentation in terms of enhancing the capacity, accomplishing seamless coverage and fulfilling the service quality requirement for a variety of users. The synergy between terrestrial infrastructure and satellite based systems would provide 'anywhere' type of communication, as satellites can reach out to places with low terrestrial infrastructure facilities. With the availability of large frequency bands of satellite, the fusion would enable robust and cost-effective communication to large volume of multimedia data. Satellites are equipped to support fixed as well as broadcast services and hence can cater a wide variety of services to a heterogeneous set of users. However, with such a synergy, we need to see the challenges also in its novel perspective. Source heterogeneity offered by multimedia traffic, user heterogeneity introduced due to various personal receiving devices and channel heterogeneities observed by these hybrid networks demand a flexible and robust communication scheme which would maneuver these heterogeneities. The signals are likely to experience fluctuations in SNR and may undergo a lot of corruption due to AWGN and fading. It requires a comprehensive effort to guarantee a certain quality of service (QoS) to multimedia signals under such diverse conditions. Heterogeneity of the network, deep fades, noise bursts encountered in the transmission path, \& mobility of the receiver are some of the reasons which would cause the received signal quality to vary significantly. Besides this, the frequency nonselective fading channel may introduce multiplicative distortion which could implicate time-varying distortion in amplitude \& a time-varying disturbance in phase. Provisioning of QoS to jittersensitive multimedia information under these conditions is specifically challenging in a broadcast environment. As it does not provide an access to channel state information and it has to address the receiving devices which differ in decoding capability, sensitivity and mobility.

In this paper, we suggest a two tier scheme which would guarantee the QoS by ensuring that the service it is not abruptly discontinued when channel SNR deteriorates. It also ascertains that slow, flat, narrowband fading does not introduce an error floor and cause BER degradation. The first tier employs multiresolution (MR) source coding to tackle source diversity offered by multimedia information and applies unequal error protection (UEP) to different resolution levels. UEP is achieved through a hierarchical (HR) constellation which constitutes of an M- QAM constellation riding over a baseline 4-PSK constellation. A more reliable transmission channel i.e. a better error protection is applied to the coarser-resolution information by mapping it to the baseline 4-PSK constellation. The finerresolution information is mapped to the M-QAM constellation. This non-uniform signal-space constellation provides different levels of protection to different resolution levels. The MR-UEP coding combination allows decoding of a single-source description at a variety of rates [1, 2, 3, and 4]. It facilitates a variety of service classes i.e. it offers a variety of error performance to the multitude of receivers. This joint source and channel coding effort of the transmitter is supported by the receiver which reduces the rate $\&$ drops the finer resolution of source information when the received SNR is found to be below a prescribed threshold. The rate is reduced by intentionally neglecting the LSBs of a symbol, i.e. by switching from 4-PSK/M-QAM hierarchical constellation to baseline 4-PSK constellation. This ensures that a coarser level of granularity is achieved in presence of large AWGN noise and deep fades. The 
intention is to moderately sacrifice the quality of reception in order to provide guaranteed QoS in adverse channel conditions.

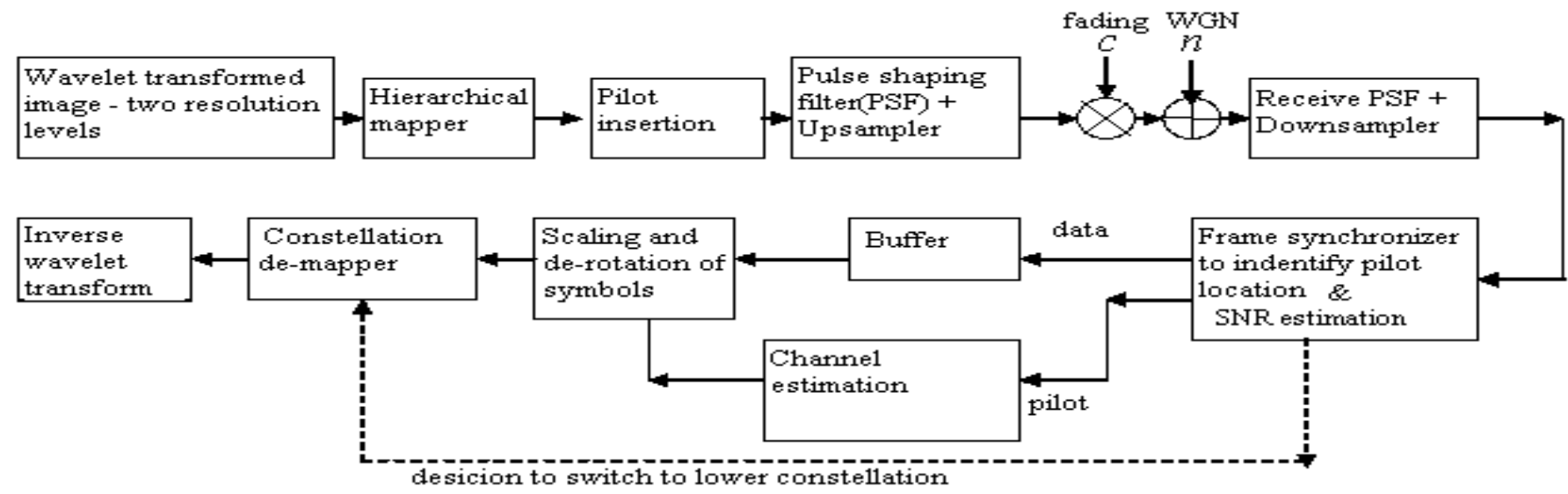

Figure 1. Baseband equivalent system model

The decision to reduce the rate by switching to a smaller constellation size is based on the estimated SNR values which are obtained at the receiver. Thus, first tier of the scheme ensures graceful degradation and enables service to heterogeneous set of receivers. Even though HR constellations aid spectrally-efficient transmission and provide UEP without additional channel coding, these constellations require a very stable reference grid for demodulation. Multiplicative corruption caused by frequency flat fading can severely degrade error performance for such embedded constellations. The second tier of the scheme works to resolve this issue. It embeds pilot symbols in the data stream at the transmitter. These pilot symbols probe the flat fading channel in a sense that it obtains the samples of the channel's complex fading envelope. At the receiver, these pilot symbols are first identified through a synchronization process. The extracted pilot symbols are then used to derive the amplitude and phase reference, which are used to compensate the effect of motion-induced phase disturbances and variations in the path gains. The process of synchronization also allows us to draw an inference regarding channel SNR, which would set the flag for rate-reduction. The open loop scheme outlined in this paper, facilitates robust transmission of multimedia data over timevarying, noisy, fading channels and caters a guaranteed QoS to a multitude of receivers in a satellite based broadcast environment.

The baseband equivalent model of the receiver is shown in Figure 1. The outline of this paper follows here. Section 1 discusses the model describing the joint algorithm of mapping resolution scalable information to HR constellation. Section 2 discusses the pilot symbol assisted coherent demodulation. The end-to-end distortions involved in the entire chain are discussed in Section 3. Simulation results are discussed in Section 4.

\section{DECODING DIVERSITY USING MR-UEP}

Multimedia information incorporates text, audio, video and image data. Among the various media types, images are the most widely used media type besides text. It is also one of the most widely used bases for representing and retrieving videos and other multimedia information. Therefore, the MR-UEP rationale described in this paper has been verified on the image information. It can be extended to other media types as well. The information, here an image, is described in a resolution scalable format. We have considered scalable description of only two resolution levels viz. coarser resolution and a finer resolution. Granularity can be improved if a description with more resolution levels is considered. A single level, separable 2-D Discrete Wavelet Transform is obtained for the image. The approximation coefficients of LL subband make coarser resolution and detail coefficients constituting of $\mathrm{HL}, \mathrm{LH} \& \mathrm{HH}$ subbands are clubbed together to make finer resolution. The coarser resolution belongs to the high protection (HP) class and the finer resolution makes low protection (LP) class. The transformed image is quantized using differential step-sizes. Even though the LL subband makes the coarser scale; in order to inflict minimum quantization error and hence to preserve highest reproduction quality, it is quantized with the finest step-size. Considering the entropy of the image, the detailed coefficients of the HH subband are quantized with same step-size as LL; while HL, LH have relatively large step-size. This variation in stepsize amongst different subbands preserves resolution scalability and provides equal length for the two data vectors representing two resolutions. The two quantized bit-streams are converted into appropriate symbols and are directly mapped to individual constellations. The HP sequence is mapped to the basic 4-PSK constellation \& the LP sequence is mapped to M-QAM constellation (here, $\mathrm{M}=16$ ) which is overriding the 4-PSK constellation. This hierarchical constellation is referred to as an MR-64-QAM in literature; here we call it HR-QAM (Figure 2). It constitutes of a linear combination of two constellation vectors; viz the constellation vector generated by baseline QPSK $\left(P_{i, q}\right)$ and, the constellation vector for M-QAM $\left(Q_{i, q}\right)$.

$$
H R_{i, q}=C 1\left(C 2\left(P_{i, q}\right)+C 3\left(Q_{i, q}\right)\right)
$$

The constants $C 1, C 2$ and $C 3$ influence the constellation expansion factor, the variance of this hierarchical constellation and the degree of protection provided to HP class and LP class of information [5 \& 6]. The values of these constants are selected in such a way that, the bit error rate of the coarse channel is lower than that of the single resolution channel. However, the bit error rate of the finer resolution channel is higher. With such an arrangement, under equal transmission power, most of the bit errors are likely to occur in the higher order coefficients. Where as, with uniform mapping, errors may occur with equal probability for all coefficients.

The probability of error for HR constellation under the influence of channel SNR is given as [7 \& 8],

$$
P_{e}=K \cdot Q\left(\sqrt{2 B T_{s y m b} \cdot \zeta_{H R} \cdot S N R}\right)
$$

$\mathrm{K}$ is the error coefficient which is determined by the average number of signals at the minimum distance. $Q($.$) is the integral$ of the tail of the unit variance Gaussian distribution, B is the transmission bandwidth $\& \mathrm{~T}_{\text {symb }}$ is the symbol period. SNR is the 
ratio of received signal power to noise power. $\zeta_{\mathrm{HR}}$ is a constellation parameter representing the ratio of the energy in the baseline 4-PSK constellation to the total average symbol energy. This ratio is a function of the constants $C 1, C 2$ and $C 3$ [5].

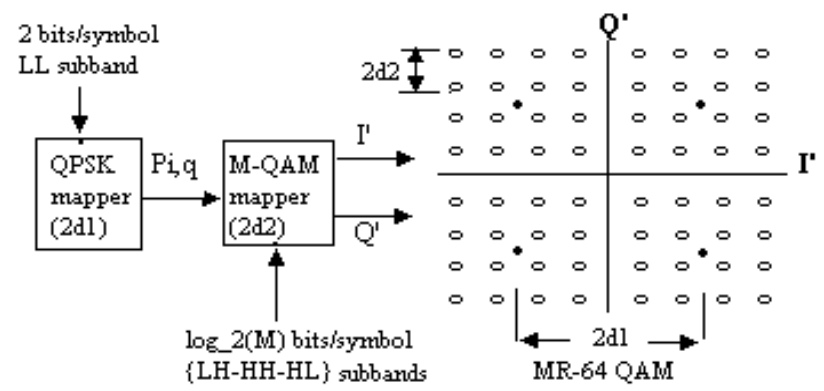

Figure 2. PSK/M-QAM mapping arrangement

Equation 2 indicates that, just like uniform constellation, performance of HR constellation is also governed by channel SNR. For a given HR constellation which is characterized by constants $C 1, C 2$ and $C 3$; probability of error increases with degrading channel quality. Under adverse channel conditions when SNR falls below an unacceptable value, we can get rid of the more corrupt part of the constellation by dropping off the over-riding constellation. Hence a certain quality of service can be achieved by reproducing only the coarser resolution which is carried by the less corrupt 4-PSK constellation. Contrarily, when SNR is high, a refined image is reproduced using both the resolutions. The reproduction quality is further improved by utilizing Pilot Symbol Assisted Modulation (PSAM) technique to guarantee QoS against slow \& flat fading.

\section{Pilot SYMbol ASSiSTed MOdUlation}

Pilot symbol assisted modulation has been discussed extensively in $[9,10,11]$. The pilot symbols are periodically inserted in the information-bearing symbols (Figure 3).The frame length $\mathrm{L}$ is chosen to be an integer multiple of the length of pilot symbol sequence $\mathrm{P}$, such that $\mathrm{L}=\mathrm{GP}$. The pilot and data symbols have the same transmit power. The channel is assumed to be frequency non-selective. This allows us to model fading as a process affecting the transmitted signal in a multiplicative form [12]. The channel is assumed to be slowly time-varying so as to model this process as a constant random variable over the entire length for which channel is being estimated. We assume perfect symbol timing recovery and carrier frequency acquisition. The complex envelope of the signal received at the output of the matched filter can be expressed as [appendix C, 13],

$$
r(k)=2 \varepsilon c(k) s(k)+n^{\prime}(k)
$$

where $\varepsilon$ is the transmitted signal energy, $n^{\prime}(k)$ is the sampled AWGN at the output of receive pulse shaping filter (PSF) with zero-mean and variance No/2 per dimension, $s(k)$ represents the complex baseband signal and pilot symbols. The sampled multiplicative distortion is of the sort of $c(k)=R(k) e^{\theta(k)+2 \Pi k T_{s y m} f_{D}}$ where $\theta(k)$ is uniformly distributed over the interval $[-\pi, \pi]$ and $f_{\mathrm{D}}$ is the Doppler frequency. $R$ represents the fading envelope having a Rayleigh distribution with unit second moment.

\subsection{Channel estimation}

The output of the matched filter is applied to the synchronizer which identifies the location of the pilot symbols. Once the location of pilots is identified, the pilots are de-multiplexed from the data and are accumulated in a separate branch (Figure 1).

\begin{tabular}{|c|c|c|c|c|c|c|c|}
\hline-- & $\mathbf{P}$ & DATA & $\mathbf{P}$ & DATA & $\mathbf{P}$ & DATA & --- \\
\hline
\end{tabular}

\section{Figure 3. Frame structure}

These accumulated pilot symbols, denoted as $S_{p}$, are used to obtain estimates of channel gain. Fading at pilot times is extracted by dividing the received signal by locally generated pilot symbols denoted as $S_{p k n,}$.

$$
\hat{c}_{p}(k)=c(k)+\frac{n^{\prime}(k)}{s_{p k n}}
$$

These estimates are interpolated to obtain fade estimates at data locations. As long as the signaling rate, $f_{s}=1 / \mathrm{T}_{\text {symb }}$, and the maximum Doppler frequency satisfy the Nyquist condition $\frac{f_{s}}{G} \geq 2 f_{D}$, the fading at data symbols can be recovered by interpolation. The interpolator uses $\mathrm{F}$ nearest pilot symbols to obtain data symbol estimates for current frame i.e. it uses $\lfloor(F-1) / 2$-pilot symbols from the previous frames and L/2 the subsequent frames. Sinc interpolator does not require apriori knowledge of channel statistics and is simple to implement. Hence we choose to use the Sinc interpolator, whose coefficients at $m^{\text {th }}$ symbol time are,

$$
h_{i}(m)=\operatorname{sinc}\left(\frac{m}{G}-i\right) ;
$$

where $i=-\left\lfloor(F-1) / 2_{-, \ldots . .,}\left\lfloor/ 2^{2}\right.\right.$. The block length over which the estimation is assumed to be constant is determined by the factor F. It influences the estimation accuracy and time lag involved in the fade correction process [9]. Complex conjugate of interpolated estimates is multiplied with the data, which has been buffered so far. This scales and de-rotates the symbols and counteracts the effect of fading. Once this correction has been implemented, BER is affected only by AWGN noise and the channel estimation error. The effect of channel estimation error is discussed further in next section.

\subsection{Pilot location indentification and SNR estimation}

The first step to obtain channel gain estimates at pilot positions is to identify the locations of the pilot symbols. An n-span differential correlator is employed for the purpose. The optimum decision rule for obtaining the pilot synchronization is to maximize the sum of two terms, the first being the usual correlation and the second being an energy correction term. This correction term is essential for fading channels, as the multiplicative distortion causes wide variation in signal strength [14 \&15]. Two sliding windows are employed. The first window is used to calculate the autocorrelation between the output of receive PSF and it's delayed version. The amount of delay is equal to the length of the pilot sequence, P. Conventionally; only one pilot symbol is inserted at every pilot location. But this does not generate sufficient decision statistics to obtain synchronization. Hence we suggest a longer pilot sequence ("all ones"). A longer sequence also helps in obtaining the synch in case of frequency offsets [16].The output of the correlator is, 


$$
y(n)=\sum_{k=0}^{L} r(n+k) \cdot r^{*}(n+k+P)
$$

The second window is used to obtain the received signal power, which is used to normalize the decision statistics. The normalized decision variable becomes independent of the instantaneous power. Hence a correction term is obtained which averages over entire frame length,

$$
b(n)=\sum_{k=0}^{L} r(n+k+P) \cdot r^{*}(n+k+P) .
$$

The decision variable is based on the normalized autocorrelation given as, $d(n)=\frac{|y(n)|}{|b(n)|}$. Gansman et. al. have proven in their subsequent work that the optimum threshold is a function of SNR. Hence output of the synchronizer can be indicative of channel SNR. If the peak falls below a prescribed value, the channel SNR can be considered poor and the rate can be adapted by switching to the lower order constellation.

\section{END-TO-END DISTORTION}

A performance measure is required that reflects tradeoff effects for MR-UEP coding, reveals the effect of fade-correction and is more relevant in context of multimedia information. Hence the performance is evaluated by observing end-to-end distortion and channel SNR. The mean value of squared error (MSD) between the original image and the reconstructed image is evaluated at various $\mathrm{E}_{\mathrm{b}} / \mathrm{No}$ values. The major contributors for end-to-end distortion are namely, the quantization noise, modulation noise due to HR mapping, effect of AWGN channel on HR constellation and channel estimation noise. The wavelet transformation is considered to be a perfectly reversible process. However, a cause of data loss could be attributed to the floatingpoint rounding-off errors, which occur while calculating forward and inverse transformations with non-integer wavelets. The other four distortions are discussed here in detail.

i) The quantization distortion: Finding the nearest reproduction vector $\boldsymbol{C}_{i}$ of the source vector $\boldsymbol{C}$ is equivalent to finding a vector that minimizes a distortion measure. Here, the Euclidean distance measures the distortion between $\boldsymbol{C}$ and $\boldsymbol{C}_{i}$ :

$$
d\left(c, \hat{c}_{i}\right)=\sum_{k=1}^{B}\left|c(k)-\hat{c}_{i}(k)\right|^{2}
$$

where $\mathrm{i}=1, \ldots, \mathrm{B}$ are the quantization levels. As mentioned in section 1, LL subband is quantized with smallest step size; hence it experiences the least quantization noise. The output of the quantizer is directly mapped to one of the constellation points. Average mapping distortion is minimzed if there exists one to one correspondence between two sets i.e. a quantization level corresponds to a modulation level. This provides very good protection against transmission errors when used with high spectrally-efficient modulations. The robustness tends to decrease when we reduce the number of states of the modulation and map more than one quantization levels to one modulation level [17].

ii) Distortion due to HR mapping: Performance degradation is observed by a QPSK receiver when it receives a hierarchical constellation. For the HR constellation, the signal power constitutes of two components: basic QPSK constellation power $\left(2 \mathrm{~d}_{1}^{2}\right) \&$ the overriding QAM constellation power $\left(2 \mathrm{~d}_{1}^{2} \lambda^{2}\right)$. The ratio of the intra-region (satellite) mapping distance $\left(2 \mathrm{~d}_{2}\right)$ and the inter-region (cloud) mapping distance $\left(2 \mathrm{~d}_{1}\right)$ is denoted as parameter $\lambda$, which is held at a constant value. The carrier power to channel noise power $(\mathrm{N})$ ratio $(\mathrm{CNR})$ of the hierarchical constellation is denoted as,

$$
C N R=\frac{2\left(1+\lambda^{2}\right) d_{1}^{2}}{N} .
$$

When an HR constellation is received by a QPSK receiver, the overriding constellation is perceived as an additional source of noise (other than channel noise $N$ ). This gives rise to scattering of constellation points which is quantified as the modulation noise ratio (MNR) [7].

$$
M N R=\frac{2 d_{1}^{2}}{N+2 \lambda^{2} d_{1}^{2}}
$$

MNR can be considered as the CNR of the hierarchical constellation as viewed by the QPSK receivers. The difference between MNR and CNR is the penalty to the QPSK receivers due to HR constellation. This penalty is described as,

$$
P_{M N R}=\frac{C N R}{M N R}=1+\lambda^{2}(1+C N R)
$$

This is the distortion added in reproduction of coarser resolution compared to its transmission as a pure QPSK constellation. Equation (11) suggests that, this penalty is a function of both $\lambda$ and CNR. For $0<\lambda<0.5, \mathrm{P}_{\mathrm{MNR}}$ varies between 1 to $(1.25+\mathrm{CNR} / 4)$, i.e. for a given $\lambda$, the penalty is higher at higher CNR.

iii) Distortion due to presence of $A W G N$ : BER for $\mathrm{HR}$ constellation under the effect of channel SNR is discussed in section 2. It reflects the contribution of AWGN noise in the distortion observed in image reproduction.

iv) Distortion due to fading-channel estimation error: Channel estimation noise prevails in case of imperfect channel estimation. The effect of estimation error on BER for M-QAM and for HR-QAM is discussed in ref. [11] \& [18] respectively. Existence of phase error even after channel compensation, causes interference amongst inphase component \& quadrature phase component. Like AWGN, amplitude estimation error affects in an additive manner [10,11]. Amplitude and phase estimation error together yield a degradation in average SNR. Performance degradation at low SNR values is mainly due to noisy initial estimate at pilot positions (second term in equation 4). Inaccuracy in interpolation introduces a noticeable error floo at high SNR values. Channel estimation noise can be reduced by improving the estimation accuracy. For a given normalized faderate, $f_{d} T_{s y m b}$, estimation can be improved by selecting a smaller block length over which the channel gain is interpolated or equivalently, keeping a higher order of interpolator and a more frequent pilot placement $[9,10$, and 11]. All of these distortions together affect the reproduction quality. Their combined effect is reflected in Mean Square of Distortion (MSD) curves which are obtained through simulations.

\section{Simulation AND RESUlts}

A single level, 2-D wavelet transform (Daubechies filter - D4 variant) is applied to a $\mathrm{R} \times \mathrm{R}$ image. The four matrices of size $\mathrm{R} / 2 \mathrm{x} / 2$ coefficients are normalized, quantized with appropriate step size and are mapped to an HR-constellation as described in section 1. Passband noise is simulated at baseband by appropriately converting $\mathrm{E}_{\mathrm{b}} / \mathrm{No}$ to $\mathrm{SNR}$ and the end to end distortion for the reproduced image is obtained. Simulations are carried out with MATLAB - R2009a. MSD is obtained by comparing the reproduced image with the source image. The transformed and quantized image is taken as the reference source 
image. If the image reproduced at the end of the receive chain is as good as this reference image, then it implies that the HR mapper, channel noise and channel estimation noise together did not produce any distortion at all. This ideal situation is indicated by zero value of MSD. As the situation parts from being ideal, the MSD curve departs from zero value.

The coarser resolution (HP class) shows relatively smaller MSD compare to the finer resolution (Figure 5, $6 \& 7$ ), as it is better protected. The values of the constants $C 1, C 2, C 3$ are adjusted (Figure 4) so that the impact of channel corruption is more prominent for LP class information. For very noisy channels, this more corrupt part of the constellation is dropped and image is reproduced with less corrupt HP class. Value of $\lambda$ is held at a constant value. Figure 4(b) shows HR constellation under the effect of static fading and AWGN. Figure 4(c) shows the constellation after scaling and derotation.

Individual MSD curves are obtained for both the resolutions for various normalized fade-rate values. Labels in the figures indicate normalized fade-rates as fdT (subscript of $\mathrm{T}_{\mathrm{symb}}$ is dropped for convenience). When the image is reconstructed using both the resolutions, then total distortion is algebraic addition of MSD values for LP and HP (Figure 7). For higher values of $\mathrm{E}_{\mathrm{b}} / \mathrm{No}$, the MSD values are constant. It implies that for higher values of $\mathrm{E}_{\mathrm{b}} / \mathrm{No}$, the distortion is mainly influenced by the mapper distortion and channel estimation noise. Figure 5(a) \& 5(b) show the results for fade correction obtained for the values of $\mathrm{G}=26$ and $\mathrm{F}=53$ for $\mathrm{HP}$ and LP class respectively. For HP class, MSD values are at par with MSD values of only AWGN (Figure 7(a)). For a given inter-pilot spacing and the interpolation order, both LP \& HP class MSD curves indicate that, as normalized fade-rates increase, channel estimation error increases and hence overall MSD increases. In order to accommodate higher fade-rates, spacing between pilots is reduced and estimation is done over smaller block lengths. These results are plotted for $\mathrm{G}=13 \& \mathrm{~F}=27$ in Figure 6 . Discussion of optimal pilot length, optimal pilot placement and interpolation order for a set of Doppler frequencies is beyond the scope of this paper. For a given fade-rate, the optimization would be between the estimation accuracy against interpolation time-lag and spectral efficiency. Longer pilot length and frequent pilot placement help to accommodate higher normalized fade-rates but effectively reduce throughput and hence affect spectral efficiency.

\section{CONCLUSION}

The proposed scheme allows a fade-corrected phase and amplitude reference for demodulation and provides guaranteed service for various channel conditions viz. fluctuating SNR and slow, narrowband, frequency-flat fading. The hierarchical constellation provides UEP to different resolutions of the source. The two constellations are combined hierarchically and the two resolution levels are respectively mapped to these constellations. The relative distances in hierarchically mapped constellations are adjusted to implicate a controlled distortion for both the resolutions. As coarser resolution is better protected, it is reproduced with comparatively less reproduction error. The end to end distortions involved in this entire two tier scheme are discussed in detail. Quantization distortion, distortion involved in mapping a secondary constellation on a baseline QPSK constellation, AWGN noise \& distortion due to channel estimation error are main contributors in the total reproduction error. The channel estimation error reflects as an additive noise and contributes in total reproduction error. For a given pilot placement and interpolation order, this distortion increases with increase in normalized fade-rate values. In order to accommodate faster normalized fade rates, the pilot symbols are to be placed more frequently and interpolation of channel gain estimates should be done over smaller block length. The results obtained through simulations prove that, for a given pilot separation and interpolation order, smaller fade-rate values produce smaller distortion. If normalized fade-rate values are sufficiently low, the distortion becomes comparable with the distortion produced by the channel undergoing only AWGN corruption.

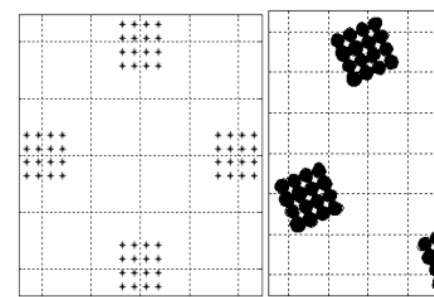

(a)

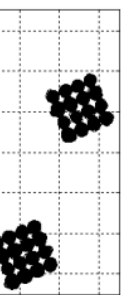

(b)

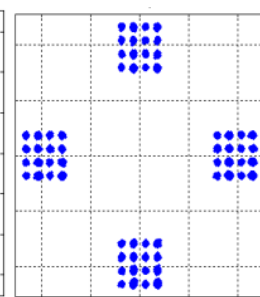

(c)
Figure 4. (a) Constellation diagram for MR-64 QAM, constellation corrupted by AWGN and static fading, (c) derotated constellation

(a) MSD for HP class, $\mathrm{G}=\mathbf{2 6}, \mathrm{F}=\mathbf{5 3}$

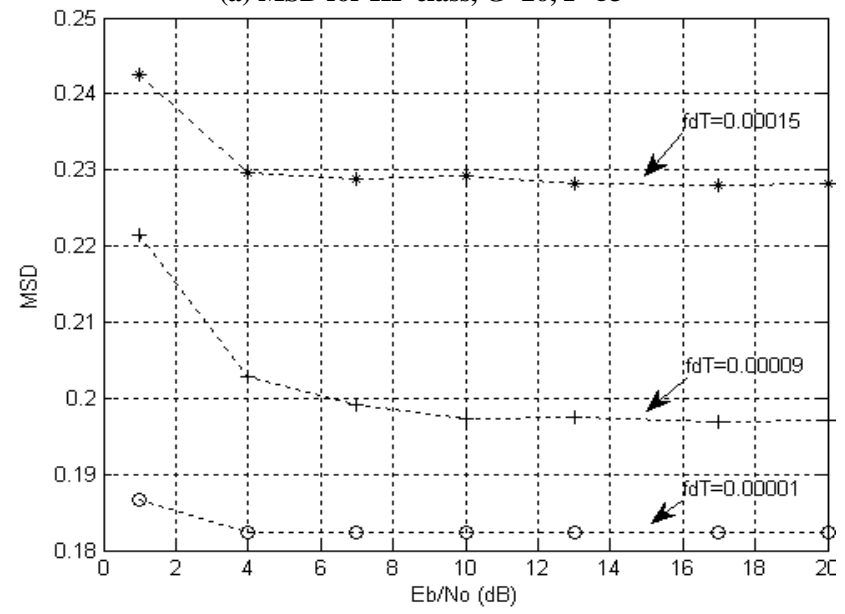

(b) MSD for LP class, $\mathrm{G}=\mathbf{2 6}, \mathrm{F}=\mathbf{5 3}$

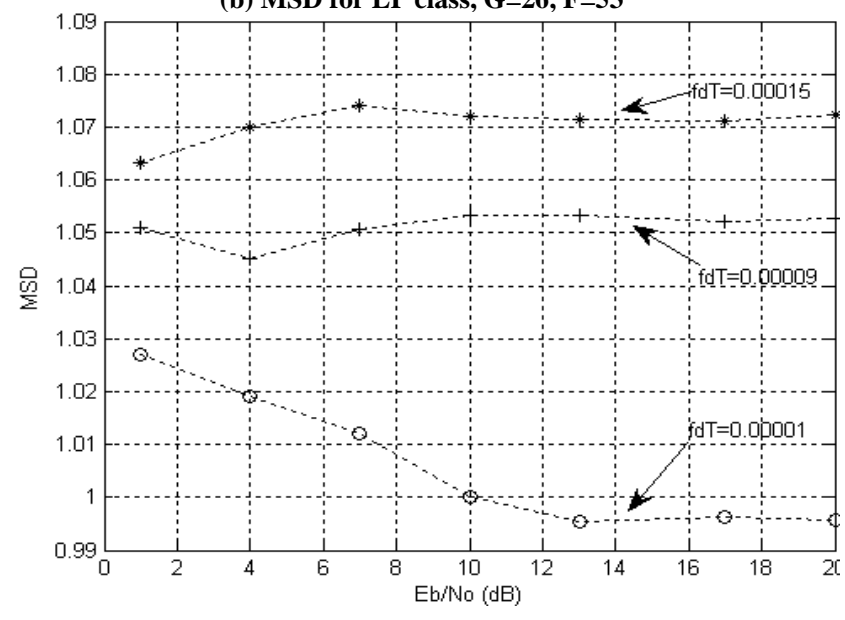

Figure 5. $M S D$ for $\mathbf{G}=\mathbf{2 6}$ and interpolation order of $\mathbf{5 3}$ for fade-rates $\mathbf{0 . 0 0 0 0 1 , 0 . 0 0 0 0 9 \& 0 . 0 0 0 1 5 ; ~ ( a ) ~ f o r ~ H P ~ c l a s s ~ i . e . ~}$ coarser resolution reconstruction, (b) for LP class-finer resolution reproduction 
(a) MSD for $\mathrm{HP}$ class, $\mathrm{G}=13, \mathrm{~F}=\mathbf{2 7}$

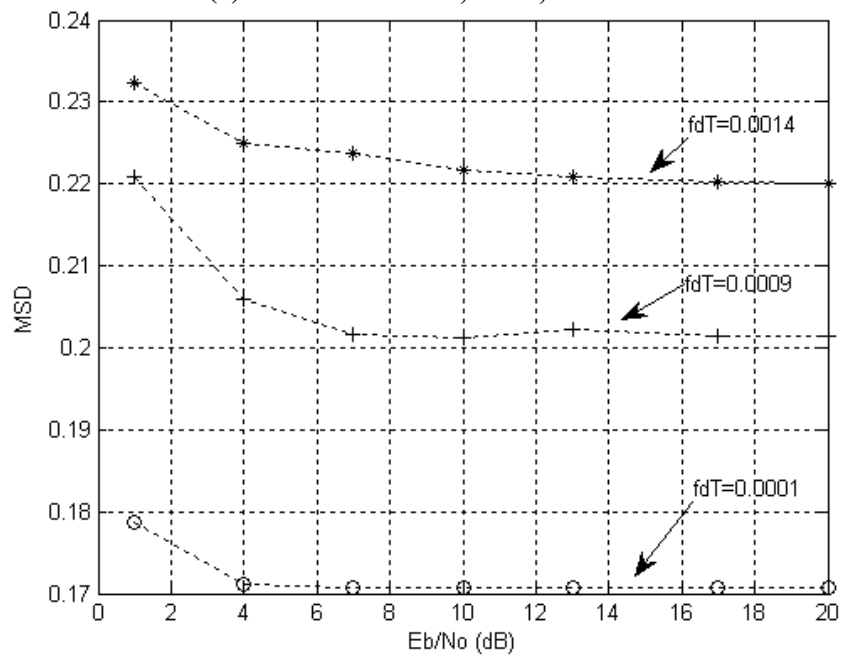

(b) MSD for $L P$ class, $G=13, F=27$

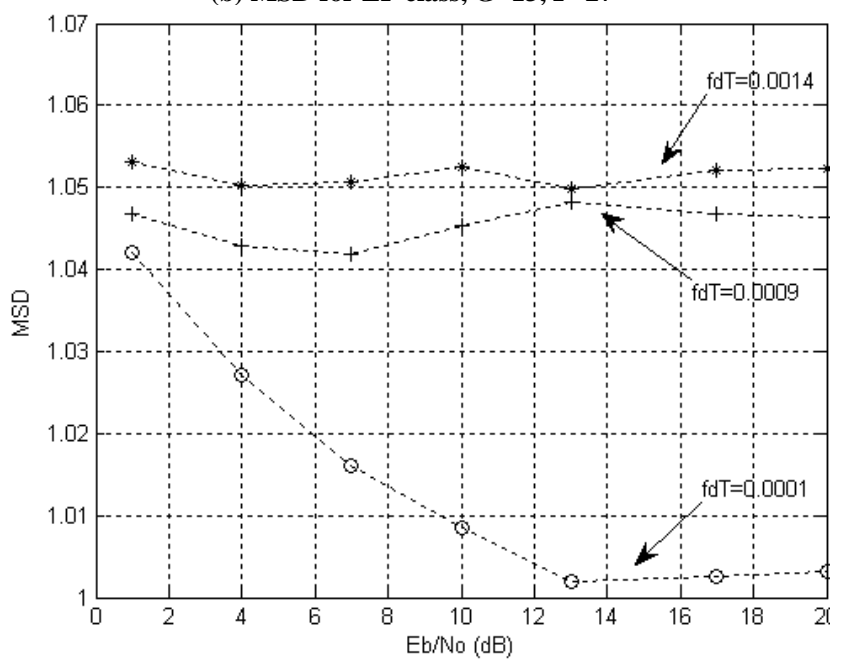

Figure 6. MSD for $\mathbf{G}=\mathbf{1 3}$ and interpolation order of $\mathbf{2 7}$ for fade rates $0.0001,0.0009 \& 0.0014$; (a) for HP class i.e. coarser resolution reconstruction, (b) for LP class-finer resolution reconstruction

(a) MSD for HP class for only AWGN channel

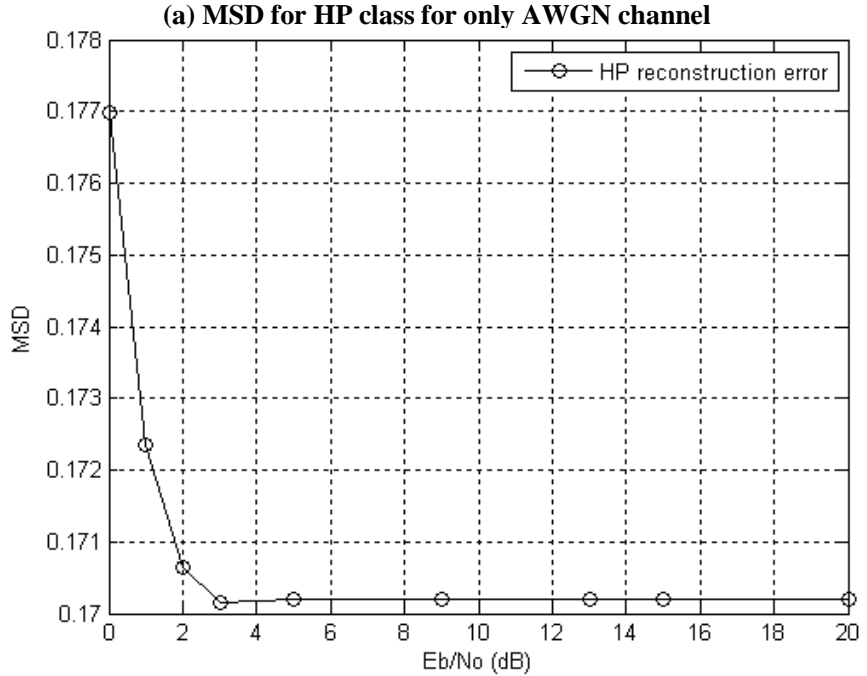

(b) MSD for LP class and entire image for only AWGN

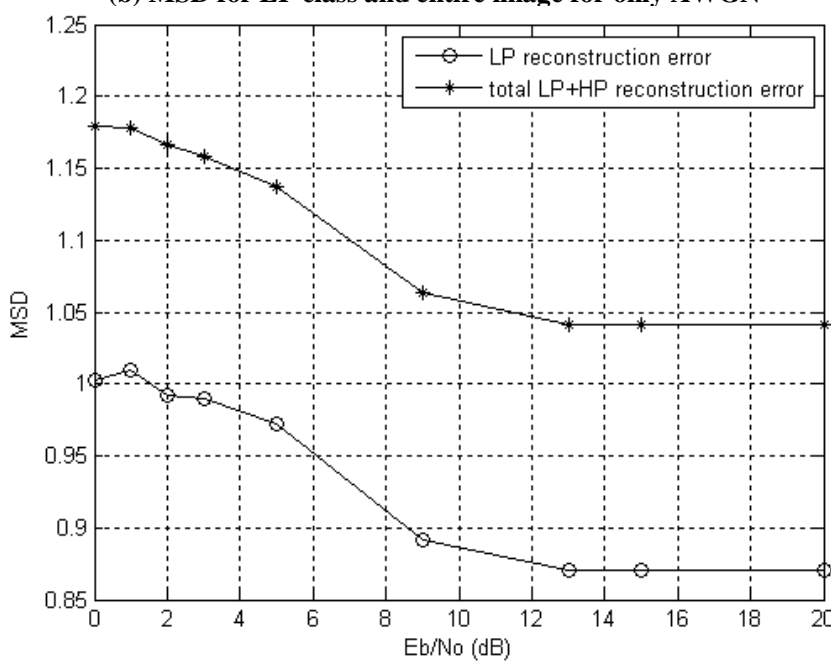

Figure 7. MSD for channel having only AWGN source-no fading; (a) for HP class; (b) MSD for LP class i.e. finer resolution and MSD for image reconstruction using both the resolutions.

\section{REFERENCES}

[1] K. Ramchandran, K. M. U. A. Ortega, and M. Vetterli, “ Multiresolution broadcast for digital HDTV using joint source/channel coding", IEEE Journal on Selected Areas Communications, vol. 11, pp. 6-23, Jan. 1993.

[2] Igor Kozintsev,Kannan Ramchandran, "Robust Image Transmission Over Energy-Constrained Time-Varying Channels Using Multiresolution Joint Source-Channel Coding”, IEEE Transactions on .Signal Procs.,vol. 46,no. 4, April 1998

[3] Khaled Fazel, Michael J. Ruf, "Combined multilevel coding and multiresolution modulation," 0-7803-0950February 1993

[4] X. Lin, L. Hanzo, R. Steele, W. T.Webb, "Subbandmultipulse Digital Audio Brodcasting for Mobile Receivers", IEEE transactions on Broadcasting, Vol. 39 No. 4 , December 1993.

[5] Pavan K. Vitthaladevuni, and Mohamed-Slim Alouini, "BER Computation of 4-M-QAM Hierarchical Constellation," IEEE Transactions on Brodcasting, vol. 47, No. 3, September 2001

[6] B. Barmada, M. M. Ghandi, , E. V. Jones, , and M Ghanbari, "Prioritized Transmission of Data Partitioned H.264 Video With Hierarchical QAM", IEEE .Signal Proc. letters, vol. 12, No.8, Aug. 05

[7] Hong Jiang, Paul A. Wildrof, "A hierarchical modulation for upgrading digital broadcast system",IEEE transactions on broadcasting, vol. 51, No.2, June 05

[8] Sorum Kotia, K. S. Dasgupta \& M. D. Desai, "Receiver based Rate Adaptation Approach through Blind SNR Estimation of Satellite Channel,", Proceedings of International MultiConference for Engineers and Computer Scientists 2010 vol. II, IMECS 2010, March 2010, ID 978-988-18210-4

[9] James K. Cavers, "An Analysis of Pilot Symbol Assisted Modulation for Rayleigh Fading Channels," IEEE 
transactions on vehicular technology, vol 40, No. 4, Nov 1991.

[10] Xiaodong Cai and Georgios B. Giannakis, "Adaptive Modulation with Adaptive Pilot Symbol Assisted Estimation and Prediction of Rapidly Fading Channels", Conference on Information Sciences and Systems, The Johns Hopkins University, March, 2003.

[11] Xiaoyi Tang, Mohamed-Salim Alouini and Andrea Goldsmith, "Effect of channel estimation error on M-QAM BER performance in Rayleigh fading, IEEE transactions on Communications, vol.47,No. 12, Dec 99.

[12] Ezio Biglieri, John Proakis, and Shlomo Shamai (Shitz), "Fading Channels: Information-Theoretic and Communications Aspects", IEEE transactions on Information Theory, Vol. 44, No. 6, October 1998.

[13] Digital communications, John. G.Proakis, Third Edition, McGraw-Hill International Editions.

[14] J. A. Gansman, M. P. Fitz, and J. V. Krogmeier, "Frame Synchronization for PSAM on Rayleigh Fading Channels," 1058-6393- IEEE Proceedings of ASILOMAR-29,1996.
[15] Dong-Uk Lee, Pansoo Kim, and Wonjin Sung, "Robust Frame Synchronization for Low Signal-to-Noise Ratio Channels Using Energy-Corrected Differential Correlation," Hindawi Publishing Corporation, EURASIP Journal on Wireless Communications and Networking Volume 2009, Article ID 345989 , doi:10.1155/2009/345989.

[16] Zae Yong Choi,\& Yong H. Lee, "Frame Synchronization in the Presence of Frequency Offset," IEEE transactions on Communications, vol.50, No 7, July 2002.

[17] Yun Li, Anthony Ephermides,"Simple rate control for Fluctuating channels in Ad-hoc wireless networks", IEEE Transactions on Communication, vol.53, No.7, July 2005.

[18] Nuno Souto, Rui Dinis, Joao C. Silva and Francisco Cercas, "Impact of Imperfect Channel estimation on the parformance of M-QAM Hierarchical constellation with Diversity",1-4244-1251-X/07, 2007. 